\title{
536.
}

\section{NOTE ON LAGRANGE'S DEMONSTRATION OF TAYLOR'S THEOREM.}

[From the Messenger of Mathematics, vol. I. (1872), pp. 22-24.]

I TAKE the occasion of the publication of the last edition of $\mathrm{Mr}$ Todhunter's Treatise on the Differential Calculus to make some remarks on the demonstration in question. $\mathrm{Mr}$ Todhunter proposes to himself to exhibit a comprehensive view of the Differential Calculus on the method of Limits; but he very properly introduces in some cases demonstrations founded upon other views of the subject, pointing out that this is the case, and explaining or indicating his objections. Thus (Chapter VI.) upon Taylor's Theorem, he remarks "Before we offer a strict demonstration of the theorem in question, we shall notice the method which it was usual to adopt in treatises on the Differential Calculus not based on the doctrine of limits," and then, after giving a demonstration depending on the relation $\frac{d}{d x} f(x+h)=\frac{d}{d h} f(x+h),\left({ }^{1}\right)$ he goes on "There are numerous objections to the method of the preceding articles, and especially the use of an infinite series, without ascertaining that it is convergent, is inadmissible; we proceed then to a rigorous investigation," which investigation (after Mr Homersham Cox) is a demonstration of the equation

$$
f(x+h)=f(x)+h f^{\prime}(x) \ldots+\frac{h^{n}}{[n} f^{n}(x)+\frac{h^{n+1}}{\mid \underline{\mid n+1}} f^{n+1}(x+\theta h),
$$

( $\theta$ between 0 and 1) whence "if the function $f^{n+1}(x+\theta h)$ is such that by making $n$ sufficiently great the term $\frac{h^{n+1}}{\mid n+1} f^{n+1}(x+\theta h)$ can be made as small as we please, then by carrying on the series

$$
f(x)+h f^{\prime}(x)+\frac{h^{2}}{\underline{2}} f^{\prime \prime}(x)+\frac{h^{3}}{[3} f^{\prime \prime \prime}(x)+\ldots
$$

1 This demonstration is similar in principle to Lagrange's but I think his is preferable; viz. the principle made use of by Lagrange is that the series has the same value whether $x$ is changed into $x+k$, or $h$ into $h+k$. 
to as many terms'as we please we obtain a result differing as little as we please from $f(x+h)$. Under these circumstances then we may assert the truth of Taylor's theorem."

I share Abel's horror of divergent series $\left({ }^{1}\right)$, and I maintain the validity of Lagrange's demonstration. When by an algebraic process we expand a function in a series, for instance the function $\frac{1}{1-x}$, by division

$$
\begin{gathered}
1-x) \frac{1}{\frac{1-x}{x}}\left(1+x+x^{2}+\& c .\right. \\
\frac{x-x^{2}}{x^{2}} \& c .
\end{gathered}
$$

in the series $1+x+x^{2}+\&$ c., and write accordingly

$$
\frac{1}{1-x}=1+x+x^{2}+\& c \text {. }
$$

all that is (or ought to be) meant is that the algebraical operations continued as far as we please will give the series of terms $1, x, x^{2}, \ldots$ or say the series of coefficients $1,1,1, \ldots$ And of course with this meaning of the equation, the objection "non constat that the series is convergent" would be wholly irrelevant, we do not say that it is, we do not care whether it is so or not. In further illustration, remark that we frequently use such an equation merely as the means of expressing the law of a series of numbers $a_{0}, a_{1}, a_{2}, \ldots$; say $a_{n}=$ coeff. $x^{n}$ in $f(x)$, where the function is assumed to be by a definite process expansible in the form $a_{0}+a_{1} x+a_{2} x^{2}+\& c$. in question. Any objection that the series is not convergent would be simply irrelevant. Now any rational or irrational algebraic function $f(x+h)$ can by ordinary algebraical processes be expanded in the form $f(x)+$ terms in $h, h^{2}$ \&c.... And if in regard to a function $f(x)$ we make the single assumption that $f(x+h)$ is expansible in a form containing powers of $h$ and reducing itself to $f(x)$ when $h$ is put $=0$, then Lagrange's demonstration shows that the powers of $h$ are $h, h^{2}, h^{3}, \& c . .$. and that the expansion in fact is

$$
f(x+h)=f(x)+h f^{\prime}(x)+\frac{h^{2}}{1.2} f^{\prime \prime}(x)+\& c . ;
$$

viz. $f(x+h)$ acquires the same value $f(x+h+k)$ whether we change therein $x$ into $x+k$ or $h$ into $h+k$; and the expression on the right-hand side is the only series in $h$ possessed of the same property. It is to be remarked that the equation contains in itself the definition of the operation of derivation, viz. the equation being true, $f^{\prime}(x)$ can only denote the coefficient of $h$ in the expansion of $f(x+h)$; and what

1 Peut-on imaginer rien de plus horrible que de débiter

$$
0=1^{n}-2^{n}+3^{n}-4^{n}+\text { etc., }
$$

$n$ étant un nombre entier positif?-QEuvres, t. II., p. 266; [Nouv. Éd., 1881, t. II., p. 257]. 
really is shown is that admitting such an operation to be possible in regard not only to $f(x)$, but to $f^{\prime}(x)$, \&c., then the coefficients $f^{\prime}(x), \frac{f^{\prime \prime}(x)}{1.2}$, \&c., are obtained from $f(x)$ by the successive repetitions of this operation and by dividing by the proper numerical denominator.

By what precedes, any objection in regard to convergency, I regard as irrelevant; and if it is said that the above-mentioned single assumption is not granted, I would either ask "What is a function"-or I would content myself with the hypothetical statement-if $f(x)$ be such that $f(x+h)$ is expansible ut suprà, then Taylor's theorem.

In regard to the demonstration given by $\mathrm{Mr}$ Todhunter, it implicitly assumes that $x$ and $h$ are both real, and (although doubtless possible) it would be considerably more difficult to find an analogous demonstration of the formula involving $f^{n+1}(x+\theta h)$ in the case of $x$ and $h$ imaginary. But the formula with the term in question is not (nor does Mr Todhunter consider it as being) Taylor's theorem; to obtain from it Taylor's theorem, we require (in the foregoing point of view) the property that $h^{n+1} f(x+\theta h)$ is expansible in a series involving $h^{n+1}$ and the higher powers of $h$, that is, the very property that $f(x+h)$ is expansible in positive powers of $h$.

Moreover admitting that the formula with the term $f^{n+1}(x+\theta h)$ is demonstrable for imaginary values of $x, h$, the formula is meaningless in the case where $x, h$ are one or both a symbol or symbols of operation: $\theta$ would certainly have no definable numerical magnitude, and if it is considered as meaning anything, then the equation in question is a mere definition of what it does mean, and ceases to be a theorem in regard to $f(x+h)$. It is impossible, in a quantitative algebra such as is presupposed in the method of limits, to put any meaning on the equation

$$
f\left(\frac{d}{d x}+h\right)=f\left(\frac{d}{d x}\right)+h f^{\prime}\left(\frac{d}{d x}\right)+\& c .
$$

which however I regard as a legitimate particular form of Taylor's theorem. 